\title{
Especificidad de la Composición del Conocimiento en las Empresas Familiares
}

\author{
Navarro de G., Ketty* \\ Villasalero, Manuel ${ }^{\star}$ \\ Donate, Mario
}

\section{Resumen}

El objetivo de este trabajo es indagar sobre la composición del conocimiento en las empresas familiares. Para ello se realizó una revisión de los trabajos publicados al respecto, tomando como referencia el modelo SECI de Nonaka y Takeuchi (1995) para el estudio del proceso de creación de conocimiento en las empresas. A partir del análisis documental, se plantean dos argumentos acerca del proceso de creación del conocimiento en las empresas familiares, basados en las características intrínsecas de las mismas. Se concluye que, considerando las características de este tipo de organizaciones, el proceso de creación del conocimiento se desarrolla de manera particular, con predominio del tipo de conocimiento tácito sobre el explícito y los modos de conversión del conocimiento internos (socialización e internalización) sobre los cruzados (externalización y combinación). Dicho conocimiento cuenta con las características pertinentes para crear valor dentro de las empresas familiares: transferibilidad, absorción, apropiabilidad y especialización.

Palabras clave: Empresa familiar, gestión del conocimiento, conocimiento, creación de conocimiento, dirección de empresas.

\section{Recibido: 20-09-12. Aceptado: 27-01-14}

* Universidad del Zulia, Instituto de Investigaciones Económicas y Sociales, Facultad de Ciencias Económicas y Sociales, Maracaibo (Venezuela), e-mail: kettydeg@gmail.com

** Universidad de Castilla La Mancha (España), Facultad de Ciencias Económicas y Jurídicas, Departamento de Organización Social, e-mail: manuel.villasalero@uclm.es

*** Universidad de Castilla La Mancha (España), Facultad de Derecho y Ciencias Sociales, Departamento de Administración de Empresas, e-mail: mariojavier.donate@uclm.es 


\title{
Specificity of the Composition of Knowledge in Family Businesses
}

\begin{abstract}
The objective of this work is to investigate the composition of knowledge in family businesses. A review of studies published on this topic was made, taking as reference the SECI model of Nonaka and Takeuchi (1995) for study of the process of knowledge creation in businesses. Starting from this documentary analysis, two arguments are proposed regarding the knowledge creation process in family businesses, based on their intrinsic characteristics. Conclusions are that, considering the characteristics of this type of organization, the knowledge creation process develops in a specific way; tacit knowledge predominates over explicit knowledge and the modes for converting internal knowledge (socialization and internalization) predominate over crossed types, such as externalization and combination. This knowledge has pertinent characteristics for creating value within family businesses: transferability, absorption, appropriability and specialization.
\end{abstract}

Keywords: Family business, knowledge management, knowledge, knowledge creation, direction of businesses.

\section{Introducción}

En el ámbito mundial existen actualmente numerosos estudios, encaminados a precisar las características intrínsecas de las empresas familiares, las cuales conforman un soporte significativo en el entramado económico de muchos países. Por otra parte, numerosas investigaciones han demostrado también el aporte fundamental de este tipo de empresa a la economía mundial y como consecuencia, al desarrollo de las sociedades (Gallo, 1991; Cabrera, 1998, Sharma, 2004). De hecho, las empresas familiares han llegado a ser la forma dominante de empresa, tanto en economías desarrolladas como en vías de desarrollo y por su aporte, tanto a la innovación y a la generación de empleos, como al producto interno bruto de las naciones, por lo que se puede esperar el aumento substancial de éstas en el futuro próximo (Sharma,
2004; Venter et al, 2005; Navarro, 2008; Hernández, 2008).

Asimismo, según investigaciones en el área, sólo el $30 \%$ de las empresas familiares en el mundo sobrevive a la segunda generación y un poco más del $14 \%$ alcanza la tercera generación (Venter et al, 2005; Vainrub y Rodríguez, 2007; Gallo, 1991). Según Lansberg (1983), Cabrera (1998), Chua et al (1999), Chua (2003), Gallo (1991) y Hernández (2008), el problema radica principalmente en la naturaleza de la empresa familiar que dificulta la separación de las relaciones familiares de las laborales; cuando la empresa está dirigida por una familia, los criterios familiares prevalecen en las decisiones gerenciales.

En este sentido, es pertinente acotar que aunque el origen de la empresa familiar se remonta a los albores de la humanidad, es recientemente cuando se ha incrementado el interés por estudiar este 
tipo de empresas, debido a su aporte a la economía mundial y a las características especiales que las diferencian del resto, por cuento son gerenciadas y controladas por sus propios dueños, quienes pertenecen a una misma familia (Chua et al, 1999; Sharma, 2004; Hernández, 2008).

La empresa familiar es una organización social de gran complejidad, ya que en ella conviven la empresa y la familia. Estos dos subsistemas no sólo se superponen sino que son interdependientes lo cual genera una gran cantidad de conflictos a superar por parte de las empresas familiares si quieren sobrevivir y posicionarse de manera exitosa en el mercado (Bañegil et al, 2011). Así, responden a una dinámica operativa especial que influye sobre su desempeño, pues en ellas interactúan dos sistemas sociales aparentemente incompatibles y solapados (Lansberg, 1983; Brenes et al, 2006; Hernández, 2008): el familiar y el empresarial. De la interrelación entre ambos sistemas emerge uno nuevo, la empresa familiar, con sinergias y fortalezas, pero también fricciones y conflictos, que no aparecían en los sistemas originales (Ayala y Navarrete, 2004).

Desde el punto de vista social, tanto la familia como la empresa, definen institucionalmente relaciones sociales en términos de un sistema único de valores, normas y principios; asimismo, cada uno tiene sus propias reglas de conducta (Venter et al, 2005; Lansberg, 1983). Estas diferencias provienen sobre todo del hecho de que cada una existe en la sociedad por razones fundamentalmente diversas. La función social primaria de la familia, por un lado, es asegurar el bienestar de sus miembros y las relaciones sociales se estructuran para satisfacer sus necesidades de desarrollo. En cambio, la razón social de la empresa es la generación de bienes y servicios a través de un comportamiento organizado de tareas (Lansberg, 1983; Rus y Rodríguez, 2005).

Sin embargo, para que una empresa sea sostenible en el tiempo como empresa familiar, en un mercado global de alta competitividad en pleno siglo XXI, debe existir una relación simbiótica y de sinergia entre la familia y la empresa. Este es uno de los grandes dilemas de la empresa familiar, por cuanto se espera que de alguna manera los resultados de la empresa generen valor para la familia y viceversa, así la creación de este valor es imposible sin la implicación de la familia (Chua et al, 2003). En este sentido, surge la gestión del conocimiento como un mecanismo para incentivar la competitividad en la empresa familiar, además de las otras ventajas que posee como herramienta gerencial.

Este trabajo representa, por tanto, una contribución al estado del arte de la gestión del conocimiento en las empresas familiares. Pese a la gran cantidad de estudios tanto teóricos como empíricos sobre las características intrínsecas de las mismas y sus efectos sobre su desempeño, son escasas las investigaciones que intentan explicar su comportamiento a través del enfoque de la gestión del conocimiento.

Se parte de los postulados del conocimiento tácito desarrollados por Nonaka y Takeuchi (1995) en sus estudios empíricos para explicar por qué la mayoría de las empresas familiares perecen antes de cumplir los cinco años de fundación. Así como también posibilita establecer el tipo de conocimiento que ha permitido a muchas empresas familiares en el ámbito mundial permanecer por más de cinco generaciones. 
¿Tendrá algo que ver con esto, la manera cómo los fundadores utilizan el conocimiento idiosincrásico o tácito para preparar a sus sucesores y en el modo cómo se convierte este conocimiento?

El conocimiento tácito se puede definir como aquel que no es visible, adquirido a través de la experiencia, presente en cualquier interrelación, proceso o acción realizada por el individuo. Es un tipo de conocimiento específico válido para quien lo posee porque está determinado por "una forma de hacer" en un contexto específico, en este caso la "manera de hacer las cosas" en el negocio familiar. Esta clase de conocimiento actúa como un pegamento que permite sentirse identificado y unido al contexto y a quienes colaboran en él (Ardichvili, 2008).

Este artículo es el resultado de una investigación documental sobre gestión del conocimiento en las empresas familiares con el propósito de indagar lo que se ha estudiado actualmente a nivel internacional, sobre la composición del conocimiento en las empresas familiares. La misma, forma parte de otra investigación de mayor envergadura que se está realizando actualmente en el estado Zulia (Venezuela), con el objetivo de explicar, entre otros, cómo se compone el conocimiento en estas empresas. Se realizó una revisión de la literatura especializada en diferentes bases de datos académicas.

\section{El Conocimiento}

El concepto de conocimiento revela que las empresas tienen diferentes tipos de conocimiento; en el ámbito de las personas: tácito-explícito, y en el ámbito de la organización: individual-organiza- cional. Cada uno de estos tipos de conocimiento proporcionan las bases para su ventaja competitiva (Clarke y Turner, 2005). También, el conocimiento es comúnmente distinguido de datos e información. Los datos representan observaciones o hechos del contexto, y por lo tanto no directamente significativos. La información resulta de colocar datos dentro de algún contexto significativo, a menudo en la forma de un mensaje.

El conocimiento es todo aquello en lo cual un individuo cree y valora, basado en la acumulación significativamente organizada de la información (mensajes) a través de la experiencia, la comunicación o la inferencia. Asimismo, el conocimiento puede ser visto, tanto como "algo" para ser almacenado como manipulado y como un proceso simultáneo de saberes e interpretación, es decir, aplicando las destrezas. Desde el punto de vista práctico, las organizaciones necesitan manejar el conocimiento tanto como un objeto, como un proceso (Zack, 1999).

El conocimiento puede ser caracterizado desde muchos puntos de vista. Las clasificaciones tradicionales distinguen entre conocimiento tácito y explícito, conocimiento general y conocimiento específico, según el contexto situacional, y conocimiento individual y colectivo (Demsetz, 1988). El conocimiento también puede ser clasificado por el tipo, incluso declarativo (conocimiento acerca de), procesal (know-how), causal (know why), condicional (know when) y relacional (know with) (Zack, 1999).

Aunque existan muchos términos para describir el conocimiento, quizás el más discutible es la distinción entre conocimiento tácito y explícito. En este senti- 
do, una diferencia más reciente que con frecuencia es citada en la literatura sobre gestión del conocimiento es proporcionada por Nonaka (1991 y 1994) y Nonaka y Takeuchi (1995), según la cual, conocimiento explícito es el conocimiento fácilmente expresado, capturado, almacenado y reutilizado. Puede ser transmitido como datos y se encuentra en bases de datos, libros, manuales y mensajes. En contraste, el conocimiento tácito es muy personal, difícil de formalizar y por lo tanto, difícil de comunicar a otros. Es profundamente arraigado en la acción y en el compromiso de un individuo de un contexto específico. El conocimiento tácito consiste en habilidades técnicas y en modelos mentales, creencias y perspectivas tan inculcadas que se dan por supuesto $y$ no se puede articular fácilmente.

Según Nonaka y Takeuchi (1995), el conocimiento nuevo en las organizaciones surge de los individuos, pero en el proceso de compartirlo es transformado en un conocimiento valioso para la organización como un todo y, por lo tanto, es posible establecer cuatro patrones para la creación de conocimiento en cualquier organización: socialización, exteriorización, combinación, e interiorización.

La socialización (de tácito a tácito) se produce cuando los individuos comparten conocimiento mediante observación, imitación y/o práctica, convirtiéndolo en su propio conocimiento tácito. Sin embargo, ninguno de ellos obtiene una visión sistemática en su conocimiento, y el mismo no puede ser utilizado por la organización como explícito.

La exteriorización (de tácito a explícito) se produce cuando un individuo es capaz de articular los fundamentos de su conocimiento tácito, lo convierte en explícito y se logra por consiguiente un crecimiento de la base de conocimiento.

La combinación (de explícito a explícito) se produce cuando un individuo combina elementos del conocimiento existente, adaptando o mostrando una nueva perspectiva del todo; sin embargo, esta forma de conocimiento no implica la expansión de la base de conocimiento de la organización.

La interiorización (de explícito a tácito) se produce cuando el conocimiento explícito es compartido por los miembros de la organización, todas las personas comienzan a utilizarlo y por lo tanto lo amplían, extienden e incorporan en su propio conocimiento tácito.

Estas cuatro fases existen en una interacción dinámica que se convierte en una espiral de conocimiento, esta última crece cada vez más a alto nivel y por lo tanto lleva al crecimiento organizacional y puede producirse de forma espontánea, pero también planificada. De allí la importancia de la gestión del conocimiento, la cual puede ser empleada en distintos tipos de organizaciones y es principalmente exitosa en aquellas en las cuales los diferentes niveles tienen un aporte significativo a la empresa. Es así como, según Nonaka (1991), se requiere una "duplicidad" en la organización la cual permite la transferencia de conocimiento, tanto tácito como explícito, a todos los niveles de esta, en un proceso de "reinvención" permanente, de emulación, mas no de competencia interna, hasta encontrar la mejor fórmula para lograrlo (López, 2011).

En este contexto, se puede sostener que el proceso de gestión del conocimiento incluye dos etapas, a saber: crear conocimiento, lo cual implica exploración, 
combinación y el descubrimiento de conocimiento mediante el hacer. Los individuos al interior de una organización crean nuevos conocimientos mediante conexiones intuitivas de las ideas existentes 0 a través de la interacción con otros individuos de la organización. Compartir conocimiento, que se produce cuando los individuos al interior de una organización transfieren y comparten el conocimiento. Al compartir este conocimiento, éste se incrementa y llega a ser más valioso, se producen sinergias de manera que el total del conocimiento alcanzado sea mayor cuantitativa y cualitativamente a la suma de los conocimientos individuales.

Un aspecto importante a considerar es que el conocimiento tácito y explícito no son entidades separadas, sino mutuamente complementarias. Éstos se relacionan el uno con el otro en las actividades creativas de los seres humanos. La interacción de estas dos formas del conocimiento es lo que Nonaka (1991) define como el proceso de conversión de conocimiento.

La literatura en gestión de conocimiento señala cuáles son las características pertinentes del conocimiento para crear valor dentro de la empresa. Estas características son señaladas por Grant (1996), a saber: transferibilidad, absorción, apropiabilidad, y especialización.

La transferibilidad se refiere a que los conocimientos deben tener la capacidad de ser transmitidos a otras personas; esto es, pueden ser captados por sus miembros y usados como un bien de la organización. Este atributo no presenta mayor problema con el conocimiento explícito, por cuanto tiene la propiedad fundamental de ser comunicado. Sin embargo, el conocimiento tácito es difícil de ser transmitido y sólo puede ser revelado a través de su aplicación. La importancia de este atributo radica en que si el conocimiento tácito no puede codificarse y sólo puede observarse a través de su aplicación y adquirido a través de la práctica, su transferencia entre las personas es lento, costoso, e incierto (Chua et al, 2003).

La visión basada en el recurso reconoce la transferibilidad de los recursos y de las capacidades de una empresa como determinante crítico de su capacidad para conferir una ventaja competitiva sostenible (Barney, 1991). Con respecto al conocimiento, la aplicación de la transferibilidad es críticamente más importante dentro de la misma empresa que entre varias empresas. Para ello es fundamental la identificación de los conocimientos tácitos y explícitos existentes en la organización.

La eficacia con la cual el conocimiento puede estar transferido depende, en parte, del conocimiento potencial para la absorción. La transferencia del conocimiento implica la transmisión y recepción. La recepción se ha analizado en términos de capacidad de absorción del recipiente (Cohen y Levinthal, 1990). Tanto en el nivel individual como de organización, la absorción del conocimiento depende de la capacidad del recipiente de agregar nuevo conocimiento al conocimiento existente. La eficacia de la absorción del conocimiento se facilita cuando el conocimiento se puede expresar en términos de un lenguaje común.

La apropiabilidad se refiere a la capacidad del propietario de un recurso para recibir un retorno igual al valor creado por ese recurso (Teece, 1986; Levin et al, 1987). El conocimiento es un recurso único, sujeto a complejos problemas de apropiabilidad. El conocimiento tácito no es directamente 
apropiable porque no puede ser transferido directamente, puede ser apropiado solamente con su uso en la actividad productiva. Por el contrario, el conocimiento explícito presenta dos problemas relacionados con la apropiabilidad. En primer lugar, como bien público, cualquiera que lo adquiera puede revenderlo sin perderlo (Arrow, 1984). En segundo lugar, el mero acto de comercializar conocimiento lo hace disponible para los compradores potenciales (Arrow, 1971). Así, a excepción de las patentes y los derechos de autor donde se protege a los creadores de conocimiento por derechos legalmente establecidos, el conocimiento es generalmente inapropriable por medio de transacciones en el mercado.

La especialización se relaciona con el principio fundamental definido por Simon (1957) según el cual la limitación del cerebro humano en la adquisición, almacenaje y procesamiento de información requiere que los individuos se especialicen en áreas particulares del conocimiento. El resultado es una mayor eficacia en la producción de conocimiento (lo cual implica la creación del nuevo conocimiento, la adquisición de conocimiento existente y el almacenamiento del mismo). Esto conduce a que los expertos sean casi invariablemente especialistas (Grant, 1996).

\section{Gestión del Conocimiento}

La idea de gestión del conocimiento permite considerar, por ejemplo, las actividades de las que se compone, explicar sus procesos de creación y transferencia o mostrar sus principales metas y objetivos, sin necesidad de definir lo que se entiende por conocimiento (Lloria,
2004). Quizá por esta razón, en la literatura es muy fácil encontrar definiciones de gestión del conocimiento abordadas desde diferentes enfoques y en ocasiones confundidos con el concepto de gestión del capital intelectual, comunidades de conocimiento o gestión de la información. También es importante destacar que se utiliza indistintamente el término administración del conocimiento para referirse a la gestión del conocimiento. Para efectos de este artículo, se asume la gestión del conocimiento como la habilidad de un ejecutivo de liderar especialistas y no especialistas y sintetizar las diferentes áreas de conocimiento de la empresa, para hacer productiva a la organización (Hernández, 2008).

Por otra parte, la gestión del conocimiento es un área compleja y heterogénea por lo que, actualmente, no existe un marco sólido consensuado acerca del conocimiento y su gestión. Las aportaciones van desde el extremo más científico hasta el más puramente divulgativo, desde el más enfocado a las tecnologías de información hasta el más preocupado por las personas, desde el más genuinamente prescriptivo en cuanto a la sistematización de políticas y decisiones concretas que debe tomar la dirección hasta el más contable, preocupado por cuantificar financieramente las diferencias entre el valor contable y de capitalización bursátil de las empresas (Oltra, 2002).

Por consiguiente, para una mejor comprensión, en los Cuadros 1 y 2 se intenta ordenar y sistematizar los distintos enfoques utilizados hasta el momento para el estudio de la gestión del conocimiento en la empresa, los cuales, de acuerdo con la bibliografía consultada, se 


\section{Enfoques de la Gestión del Conocimiento: Dirección Estratégica}

\begin{tabular}{|c|c|c|c|c|}
\hline \multirow[t]{2}{*}{ Teoría } & \multirow[t]{2}{*}{ Concepción } & \multicolumn{3}{|c|}{ Enfoques que comprende } \\
\hline & & Enfoque & Fundamento Básico & $\begin{array}{l}\text { Principales } \\
\text { Referencias }\end{array}$ \\
\hline \multirow[t]{4}{*}{$\begin{array}{l}\text { Dirección } \\
\text { Estratégica }\end{array}$} & $\begin{array}{l}\text { La gestión del } \\
\text { conocimiento } \\
\text { como una } \\
\text { dimensión de } \\
\text { la estrategia } \\
\text { competitiva }\end{array}$ & Recursos & $\begin{array}{l}\text { Las variaciones en los } \\
\text { resultados de las } \\
\text { empresas que compiten } \\
\text { en una misma industria } \\
\text { pueden explicarse a partir } \\
\text { de las diferencias en su } \\
\text { dotación de recursos }\end{array}$ & $\begin{array}{l}\text { Wernerfelt (1984) } \\
\text { Barney (1991) } \\
\text { Peteraf (1993) } \\
\text { Barney y Arikan } \\
\text { (2001) } \\
\text { Grant (1996 y 1997) } \\
\text { Mahoney y Pandian } \\
\text { (1992) }\end{array}$ \\
\hline & & $\begin{array}{l}\text { Capacidades } \\
\text { Dinámicas }\end{array}$ & $\begin{array}{l}\text { Las capacidades dinámicas } \\
\text { son las rutinas organizativas } \\
\text { y estratégicas por las que } \\
\text { los directivos alteran su base } \\
\text { de recursos, adquieren y } \\
\text { distribuyen } \\
\text { los recursos, los integran } \\
\text { y recombinan para generar } \\
\text { nuevas estrategias de } \\
\text { creación de valor. }\end{array}$ & $\begin{array}{l}\text { Teece, Pisano y } \\
\text { Shuen (1997) } \\
\text { Grant (1996) } \\
\text { Kogut y Zander (1992) } \\
\text { Eisenhardt y Martin } \\
\text { (2000) } \\
\text { Henderson y } \\
\text { Cockburn (1994) }\end{array}$ \\
\hline & & Competencias & $\begin{array}{l}\text { Los recursos de una } \\
\text { empresa se componen } \\
\text { de factores o activos y } \\
\text { se agregan en competencias } \\
\text { esenciales. }\end{array}$ & $\begin{array}{l}\text { Prahalad y Hamel } \\
\text { (1990) } \\
\text { Spender y Grant } \\
\text { (1996) } \\
\text { Henderson y } \\
\text { Cockburn (1994) }\end{array}$ \\
\hline & & Conocimiento & $\begin{array}{l}\text { Las diferencias de resultado } \\
\text { entre empresas son } \\
\text { consecuencia de sus } \\
\text { diferentes bases de } \\
\text { conocimiento y capacidades, } \\
\text { las cuales difieren en el } \\
\text { conocimiento que desarrollan } \\
\text { y utilizan }\end{array}$ & $\begin{array}{l}\text { Grant (1996) } \\
\text { Bierly y Chakrabarti } \\
(1996)\end{array}$ \\
\hline
\end{tabular}

Fuente: Elaboración propia a partir de la revisión de literatura. 


\section{Enfoques de la Gestión del Conocimiento: Teoría de la Organización}

\begin{tabular}{|c|c|c|c|c|}
\hline \multirow[t]{2}{*}{ Teoría } & \multirow[t]{2}{*}{ Concepción } & \multicolumn{3}{|c|}{ Enfoques que comprende } \\
\hline & & Enfoque & Fundamento Básico & $\begin{array}{l}\text { Principales } \\
\text { Referencias }\end{array}$ \\
\hline \multirow[t]{3}{*}{$\begin{array}{l}\text { Teoría de la } \\
\text { Organización }\end{array}$} & $\begin{array}{l}\text { Centra su atención } \\
\text { en la creación de } \\
\text { conocimiento en el } \\
\text { interior de las } \\
\text { organizaciones, } \\
\text { estudiando la } \\
\text { naturaleza activa y } \\
\text { subjetiva del } \\
\text { conocimiento, } \\
\text { representada por } \\
\text { términos tales como } \\
\text { compromiso y } \\
\text { creencias, que } \\
\text { están } \\
\text { profundamente } \\
\text { enraizadas en }\end{array}$ & $\begin{array}{l}\text { Aprendizaje } \\
\text { Organizativo }\end{array}$ & $\begin{array}{l}\text { Ninguna organización } \\
\text { puede dirigirse o } \\
\text { entenderse a partir de un } \\
\text { vacío cognitivo, sino, que } \\
\text { se parte de una o varias } \\
\text { teorías sobre la acción, } \\
\text { es decir, de un conjunto } \\
\text { de creencias, estrategias, } \\
\text { supuestos o modelos sobre } \\
\text { el comportamiento humano } \\
\text { intencional de los que se } \\
\text { postula una cierta validez } \\
\text { general }\end{array}$ & $\begin{array}{l}\text { Argyris y Schön (1978) } \\
\text { Bueno (2002) } \\
\text { Senge (1990) } \\
\text { Lloria (2004) } \\
\text { Williams (2001) } \\
\text { Kim (1993) }\end{array}$ \\
\hline & $\begin{array}{l}\text { los sistemas de } \\
\text { valores individuales } \\
\text { y organizativos }\end{array}$ & $\begin{array}{l}\text { Enfoque } \\
\text { Cognoscitivo }\end{array}$ & $\begin{array}{l}\text { El ambiente organizativo } \\
\text { no es una realidad objetiva, } \\
\text { sino que, por el contrario, } \\
\text { constituye una } \\
\text { representación subjetiva } \\
\text { en la mente del decisor. }\end{array}$ & $\begin{array}{l}\text { Weick (1991) } \\
\text { Nonaka (1991) } \\
\text { Nonaka y Tackeuchi (1995) }\end{array}$ \\
\hline & & $\begin{array}{l}\text { Enfoque } \\
\text { Evolucionista }\end{array}$ & $\begin{array}{l}\text { Se estudia la evolución } \\
\text { de las instituciones y del } \\
\text { marco económico como } \\
\text { consecuencia de factores } \\
\text { de cambio que tienen su } \\
\text { origen en procesos de } \\
\text { selección y posibles } \\
\text { mutaciones }\end{array}$ & $\begin{array}{l}\text { Langlois y Everett (1984) } \\
\text { (Nelson y Winter (1982) }\end{array}$ \\
\hline
\end{tabular}

Fuente: Elaboración propia a partir de la revisión de literatura.

agrupan en dos grandes campos de pensamiento: la dirección estratégica de la empresa y la teoría de la organización.

\section{La Empresa Familiar}

Sobre el concepto de empresa familiar no existe un consenso entre la comunidad científica sobre cuáles son los parámetros que lo delimitan (Barroso, 2013) y cuyo debate sigue abierto después de décadas estudiando e investigando este tipo de empresas. Lo cierto es que la mayoría de los autores identifican los siguientes aspectos como dimensiones principales presentes en este tipo de organizaciones (Lansberg, 1983; Handler, 1989; Henry, 2012): la participación de la familia en la propiedad y/o en la dirección de la empresa, la interdependencia de las variables propiedad y dirección. Es así como se pueden encontrar muchas definiciones 
diferentes de empresa familiar. En este sentido, Habbershon y Williams (1999), encontraron 44 definiciones distintas sobre la materia. El punto común de todas las definiciones es la implicación de la familia en el negocio. En lo que respecta a todo lo demás, la empresa familiar tiene las mismas características de cualquier otro tipo de organización (Rus y Rodríguez, 2005).

En este sentido, para efectos de este trabajo, una empresa familiar es aquella que presenta tres condiciones: (a) los miembros de la familia propietaria la controlan por varios medios; (b) ellos ejercen el control sobre la alta dirección; y (c) los miembros de la familia propietaria son sucesores o piensan en ser sucesores del negocio (Suehiro y Wailerdsak, 2004), y desean que la empresa continúe en el futuro en manos de sus descendientes.

A diferencia del resto de las organizaciones, en las familiares se identifican dos sistemas: la familia y el negocio. Las familias están conectadas por lazos emocionales, internamente orientadas y consideran la lealtad y el cuidado de los miembros de la familia como valores importantes. El sistema familia intenta limitar el cambio y mantener el equilibrio dentro de la familia. Por otro lado, el sistema empresa se centra en la realización de tareas y se orienta hacia el exterior. Este sistema tiene como objetivo producir bienes y servicios para el mercado con el fin de obtener beneficios. A diferencia del sistema familia, este utiliza el cambio para reaccionar con eficacia a las alteraciones en el entorno (Barroso, 2013).

En una empresa no familiar estos sistemas están presentes, pero funcio- nan por separado el uno del otro, mientras que en una empresa familiar, no sólo se superponen o interactúan, sino que también son dependientes el uno del otro. Aunque ambos sistemas tienen objetivos opuestos que pueden causar tensiones específicas, cuando están equilibrados, la interacción puede proporcionar beneficios, dando a estas empresas una ventaja competitiva única sobre las no familiares (Lansberg, 1983; Barroso, 2013).

Tales particularidades conducen a inferir que en cuanto al modo de gestionar el conocimiento, las empresas familiares pudieran comportarse de manera diferente a las no familiares. Esto se evidencia en las numerosas investigaciones realizadas. Diferentes autores verifican que, por ejemplo desde el punto de vista organizativo, las empresas controladas por familias tienen un proceso de toma de decisiones más centralizado y sus sistemas de control son menos formalizados (Morris et al, 1997; Venter et al, 2005; Navarro, 2008), debido a la influencia de la familia en el negocio, situación que contradice los postulados teóricos sobre la organización empresarial y la toma de decisiones. Esta es apenas una de las razones por la cual se plantea la gestión del conocimiento como un enfoque válido para decodificar aquellos conocimientos que hacen a una empresa familiar competitiva y cómo hacerla más sostenible.

A continuación se discuten varios planteamientos en torno a la gestión del conocimiento y la empresa familiar, esbozándose al final dos proposiciones concluyentes en torno al tema. 


\section{Especificidad de la composición del conocimiento en la empresa familiar}

En la literatura sobre el tema se describe al conocimiento tácito como aquel que reside en el interior de los individuos y es difícil de transmitir y codificar y, además, es dependiente del contexto. Es decir, el grado en el que el conocimiento es integrado en un contexto específico, determina su dependencia, el cual ha sido utilizado para transferir más eficazmente el conocimiento. Por ello, un contexto relevante y familiar ayuda a la transferencia de conocimiento (Endres et al, 2007).

La generación de conocimiento, la gestión del conocimiento y la transferencia de conocimiento desde el fundador hacia el sucesor son importantes para el éxito de la sucesión de la empresa. Aunque este conocimiento puede tener dos formas, explícita y tácita, la naturaleza del conocimiento como parte de la familiness ${ }^{1}$ de una empresa es principalmente tácito. Este conocimiento tácito a menudo permanece en el propietario y otras personas clave en el negocio, es significativo para el desarrollo y mantenimiento de la ventaja competitiva de la empresa, y es más importante para la empresa familiar que para las no familiares (Cabrera, 1998). En este sentido, el conocimiento tácito arraigado en el fundador es un activo estratégico y es más efectivamente transferido en las empresas familiares que en las no familiares (Henry, 2012).

Por consiguiente, una de las claves de supervivencia de la empresa familiar es la transferencia de conocimientos entre los miembros de la familia y los cambios que las nuevas generaciones deben llevar a cabo para adaptarse. El conocimiento tácito puede perderse si no se comparte y se traspasa de una generación a otra. Este conocimiento puede ser más fácilmente compartido dentro de las empresas familiares debido a las características especiales que las diferencian de las empresas no familiares. En la empresa familiar, los sucesores necesitan adquirir el conocimiento de la generación anterior, pero también han de añadir el conocimiento nuevo adquirido a través de su educación y de su experiencia personal (Barroso, 2013).

Este conocimiento está arraigado en los valores y la cultura de la familia y la empresa y es a menudo transmitido a través de historias y comunicaciones informales que suceden mediante un proceso donde se completa la transferencia de conocimiento tácito, y el éxito de esta transferencia es significativamente determinado por la relación entre el propietario-fundador y el sucesor. Las relaciones de confianza contribuyen al éxito de la empresa familiar, a la transferencia de conocimientos y a una sucesión exitosa (Henry, 2012). Es así como, compartir conocimientos entre los miembros es necesario para la empresa familiar, pues unas relaciones cordiales y prósperas en la familia

Familiness es un concepto exclusivamente relacionado con la investigación en empresa familiar, definiéndose como el conjunto de recursos propios de una empresa como resultado de la implicación de la familia en la misma. 
son requisitos previos para el éxito y longevidad de la empresa (Barroso, 2013).

Estos planteamientos permiten formular un primer argumento que se propone a manera de hipótesis, como una contribución al análisis y comprensión del proceso de creación de conocimiento en las empresas familiares:

Argumento 1: Las empresas familiares utilizan una mayor proporción de conocimiento tácito que de conocimiento explícito.

Este argumento se formula bajo dos premisas: la primera es que está centrada en el nivel directivo, vale decir, cuando la gestión de la empresa está en manos de la propiedad. La segunda es que no se formula en términos de una comparación externa con las empresas no familiares, sino como una capacidad interna dentro del grupo de empresas familiares, referida a la composición del conocimiento que emplean.
En concordancia con este planteamiento, Nonaka (1991) y Nonaka y Takeuchi (1995), asumen la creación de conocimiento como una espiral que se inicia a partir de las certezas e intuiciones individuales. Luego, en un proceso de conversión que involucra diferentes modos visualizados como etapas (socialización, externalización, combinación e internalización), este conocimiento tácito se convierte en explícito, para luego evolucionar hacia nuevo conocimiento tácito (Figura 1).

En el caso de las empresas familiares, el proceso de creación de conocimiento se puede percibir en dos instancias: la familia y la empresa. En la instancia familiar, la transferencia de conocimiento se produce durante las etapas de socialización de la familia, que comienza a una edad muy joven en el entorno familiar, y la socialización de la empresa, iniciada

Figura 1. Espiral de Creación de Conocimiento

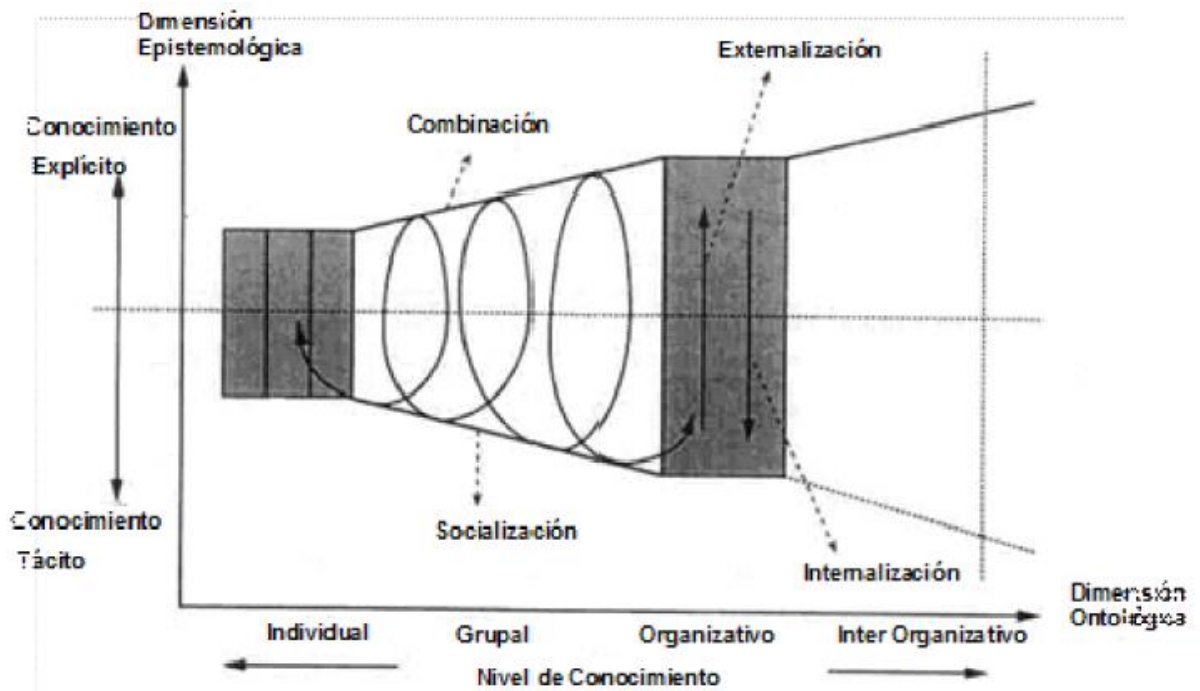

Fuente: Nonaka y Takeuchi (1995). 
una vez que el sucesor se dedica al negocio y ha sido identificado como tal (García et al, 2002). En la instancia empresa, la transferencia de conocimiento está marcada por el elevado grado de compromiso, responsabilidad y dedicación hacia la organización, tanto por parte de los miembros de la familia, como por el resto de la fuerza laboral, cuyo comportamiento es más entusiasta y se siente parte del equipo (Gallo, 1991), así como por sus propias formas de "hacer las cosas", una tecnología especial o un know-how comercial que no poseen los competidores.

Por tanto, esta capacidad debe extenderse a través de todos los miembros de la familia, con el fin de experimentar y desarrollar nuevos sistemas de captura y construcción del conocimiento, así como experiencias acumuladas por los mismos (Comeche, 2007). Es así como el conocimiento en la empresa familiar incluye la información contextual, la experiencia enmarcada, las creencias, los valores, la percepción experto, el know-how y las habilidades para realizar tareas.

Este argumento es una de las razones por la cual Cabrera (1998) y Henry (2012) sostienen que el conocimiento tácito ocupa un papel central en el desarrollo de una ventaja competitiva sostenible en las empresas familiares. Así, es precisamente, esta noción de conocimiento tácito e información no codificable la que confiere a los activos intangibles gran parte de su valor estratégico, pues le concede su carácter de imitabilidad y sustituibilidad imperfecta (Peteraf, 1993). Estas características, unidas al concepto de orgullo asociado a la empresa familiar, están integradas en la idea de "reputación" considerada por Grant (1991) como otro de los recursos intangibles fuente de ventaja competitiva presentes en las empresas familiares.

Estos recursos socialmente complejos pueden ser valiosos en el sentido de que permiten a estas organizaciones formular e implementar estrategias como las basadas en atender segmentos de mercados concretos, en priorizar la calidad del producto o servicio, o en una elevada velocidad de respuesta del cliente. Son a menudo difíciles de imitar; sus efectos estratégicos sobre la organización tienen un carácter de "indivisibilidad" en la medida en que reflejan la historia única de una organización y las habilidades directivas necesarias para gestionarlos son difícilmente imitables (Cabrera, 1998).

En tal sentido, la empresa familiar trabaja y se diversifica sobre la base de lo que tradicionalmente se ha hecho bien, apoyándose en un alto grado de conocimiento sobre cómo actuar en determinados mercados, con clientes específicos o con productos o servicios concretos. Para ello cuenta con cada uno de sus directivos, además, con oportunidades históricas particulares a explotar y con los valores y creencias únicas de los fundadores, para capacitar a la organización y a sus sucesores, así como para concebir, elegir e implementar las estrategias como una oportunidad para su competitividad (Barney, 1991). 


\section{Modos de conversión del conocimiento en la empresa familiar}

El modelo de creación del conocimiento de Nonaka y Takeuchi (1995) descansa en dos dimensiones: una dimensión epistemológica que distingue entre conocimiento tácito y explícito y una dimensión ontológica que distingue entre cuatro niveles de agentes creadores de conocimiento: individuo, grupo, organización y nivel interorganizativo.

Según Nonaka y Takeuchi (1995), la creación de conocimiento es un proceso de interacciones entre el conocimiento tácito y el conocimiento explícito. Este proceso dinámico está anclado en la presunción de que el conocimiento humano es creado y expandido a través de la interacción social entre conocimiento tácito y explícito. El conocimiento es, entonces, un recurso de gran valor en la empresa, con un importante potencial de generación de rentas a largo plazo, cuya transferibilidad es compleja (Kogut y Zander, 1992), es difícil de agregar en ocasiones y es apropiable sólo en la medida en que ha sido socializado (Grant, 1996).

Mientras cada uno de los cuatro modos de conversión de conocimiento (socialización, externalización, internalización y combinación) puede crear nuevo conocimiento de manera independiente, el tema central del modelo de creación de conocimiento organizativo viene dado por la interacción dinámica entre los diferentes modos de conversión de conocimiento.

Para comprender mejor cómo se lleva a cabo este proceso en el ámbito de las empresas familiares se toma como base las aportaciones de Barroso (2013), al ciclo SECI de creación de conocimiento propuesto originalmente por Nonaka y Tackeuchi (1995), a saber:

a. Socialización (de tácito a tácito): es el proceso de adquirir conocimiento tácito a través de compartir experiencias por medio de exposiciones orales, documentos, manuales y tradiciones, añadiendo el conocimiento novedoso a la base colectiva de la organización. La socialización es la vía predominante para transferir conocimiento desde el propietario a los empleados y entre los propios empleados. Un proceso fundamental de la empresa familiar es la experiencia previa, que es un aspecto imprescindible para la transmisión de conocimiento tácito. El aprendizaje que efectúan los miembros de la familia desde niños mediante la observación y el conocimiento transmitido de generación a generación, ilustra la importancia que tiene la socialización como fuente de transmisión de conocimientos en la empresa familiar. De este modo, cuando llega el momento de incorporarse a la empresa, poseen un gran conocimiento del negocio.

b. Exteriorización (de tácito a explícito): es el proceso de convertir conocimiento tácito en conceptos explícitos. Supone hacer tangible, mediante el uso de símbolos y códigos lingüísticos y metáforas, conocimiento de por sí difícil de comunicar, integrándolo en le cultura de la organización. Es la actividad esencial en la creación de conocimiento porque si se da la transferencia de conocimiento se producirá de una manera más eficiente y eficaz, pues su propia naturaleza de ex- 
plícito la hace más fácil de capturar y utilizar. Sin embargo, debido principalmente a la cultura organizacional de no compartir lo que sabe, a la empresa familiar le es difícil entrar en esta dinámica, pues los fundadores no están muy dispuestos a explicitar sus fortalezas personales, por pensar que esto haría que dejasen de ser imprescindibles para la empresa.

c. Combinación (de explícito a explícito): Es el proceso de crear conocimiento explícito al reunir conocimiento explícito. Estos conocimientos provienen de diversas fuentes, mediante el intercambio de conversaciones telefónicas, las redes sociales, reuniones, correos, entre otros, y se pueden categorizar, confrontar y clasificar para formar bases de datos que produzcan conocimiento explícito; no obstante, esta combinación no amplía realmente el conocimiento existente en la empresa. En la empresa familiar este conocimiento no es tan visible como otras formas de conocimiento, pero la existencia de encuentros donde discutir problemas de la empresa como la asamblea familiar o el consejo de familia, pueden permitir de alguna forma la combinación de conocimientos.

d. Interiorización (de explícito a tácito): es el proceso de incorporación de conocimiento explícito en conocimiento tácito. La empresa, incorporando el nuevo conocimiento explícito al que ya poseía, intentará llevar a cabo nuevas formas de búsqueda y experimentación, generando nueva experiencia y nuevo conocimiento tácito. Más allá de ser una labor de adquisi- ción de conocimiento basada en el análisis, se entra en una etapa de aprendizaje a través de un refinamiento continuo de la experiencia. En el ámbito de la empresa familiar, el proceso de formación y su implicación en la empresa, tanto a miembros familiares como no familiares, les permiten hacer suyos los conocimientos de la organización, incorporándose el conocimiento explícito de la misma en la base de los conocimientos tácitos de los miembros. La sucesión es un claro ejemplo de conversión de conocimiento tácito en tácito.

Los planteamientos anteriores permiten formular el segundo argumento:

Argumento 2: En las empresas familiares, en la relación entre el grupo familiar y el resto de la empresa, dominan los modos de conversión de conocimiento interno sobre los modos de conversión del conocimiento cruzado.

En el Cuadro 3 se muestra un ejemplo de cómo funciona el ciclo SECI en las empresas familiares.

El ciclo SECl ayuda a comprender la generación, transferencia y aplicación de conocimientos en las empresas familiares. Pero también es necesario añadir que gran parte de este conocimiento está concentrado en el nivel individuo (fundador) y en el nivel grupo (familiar). Es decir, el conocimiento tácito del fundador-emprendedor de la empresa y del personal ubicado en puestos clave de la empresa, generalmente miembros de la familia, no cumple un ciclo regular de conversión del conocimiento, por cuanto el mismo no se difunde en toda la organización. Ello forma parte de una cultura organizativa cuyo objetivo es el de conseguir la estabilidad del 


\section{Cuadro 3}

Ciclo SECI en las Empresas Familiares

\begin{tabular}{ccc}
\hline & $\begin{array}{c}\text { Conocimiento } \\
\text { tácito }\end{array}$ & $\begin{array}{c}\text { Conocimiento } \\
\text { explícito }\end{array}$ \\
\hline Conocimiento tácito & $\begin{array}{c}\text { Socialización } \\
\text { Sucesión }\end{array}$ & $\begin{array}{c}\text { Exteriorización } \\
\text { Protocolo }\end{array}$ \\
\hline Conocimiento explícito & Interiorización & Combinación \\
& Gestión de & Organos de gobierno \\
\hline
\end{tabular}

Fuente: Barroso (2013).

negocio y el afianzamiento de la reputación familiar, por lo que el control de la información se convierte en una herramienta básica (García, 2001). En consecuencia, es necesaria la transformación del conocimiento a nivel organizacional para convertirse en la base de la capacidad organizativa, de tal manera que el conocimiento adquirido por la nueva generación sea capaz de crear mayores ventajas competitivas.

Para los efectos, se denomina modos de conversión internos a aquellos en los cuales no se da la interacción de conocimiento tácito a conocimiento explícito (socialización e internalización). Los modos de conversión cruzados son aquellos donde no existe la interacción de conocimiento explícito a tácito (combinación y externalización).

La formulación de este argumento descansa sobre la naturaleza de la estructura organizativa en tales empresas y las relaciones establecidas en la misma. En efecto, la centralización de las decisiones y la poca formalidad de los controles, normas y procedimientos, llevan a inferir que los mecanismos de comunicación dominantes son los de cara a cara, debido a la falta de intención por parte del grupo familiar o del fundador de hacer explícitos todos los conocimientos de la empresa. Este hecho establece una barrera de creación conocimiento organizativo que conlleva a la permanencia del conocimiento tácito entre el grupo familiar y la intrascendencia hacia el resto de la organización, recreando un proceso de creación de conocimiento diferente entre el grupo directivo y el resto de la organización.

Por último, se puede señalar que el conocimiento de la organización es resultado de los conocimientos de los agentes individuales que pertenecen a ella. La mera posesión de conocimiento por parte de algunos miembros de la empresa no garantiza una gestión adecuada y dicha gestión, para que sea adecuada, debe estar a disposición de diversos agentes en las mejores condiciones.

\section{Conclusiones}

El trabajo documental y análisis conceptual realizado permiten una aproximación al cómo entender el proceso de creación de conocimiento en la empresa familiar. En primer lugar se infiere que el proceso de creación del conocimiento en este tipo de empresas se desarrolla de 
manera particular, por las características especiales de las mismas, predominando el tipo de conocimiento tácito frente al explícito y los modos de conversión del conocimiento internos (socialización e internalización), frente a los cruzados (externalización y combinación).

Asimismo, también se puede apreciar que en el caso de las empresas familiares, el conocimiento cumple con las características señaladas por Grant (1996), de transferibilidad, absorción, apropiabilidad y especialización.

La transferibilidad, aun cuando los argumentos esgrimen una concentración de conocimiento clave sobre el negocio en el núcleo familiar y además, de un predominio del conocimiento tácito sobre el explícito, el poder ser transmitido del fundador a otros integrantes de la familia o de una familiar no fundador a otro. Brinda la oportunidad de ser usado como un bien de la organización, aunque con la particularidad de que generalmente su adquisición y aplicación se observa a través de la práctica (por ejemplo, cuando los niños aprenden sobre el negocio de sus padres), por lo cual se torna lento y puede tardar años, hasta que el sucesor se incorpora a la dirección de la empresa.

En el caso de la absorción, el conocimiento en las empresas familiares adquiere esta característica y se facilita, en primer lugar, debido a la afinidad de intereses y la confianza permiten un lenguaje común que los identifica con familia y organización, y también porque el propietario transfiere al sucesor y al resto de la organización aquel conocimiento considerado conveniente de transferir y éstos son capaces de crear nuevo conocimiento.
Con relación a la apropiabilidad en las empresas familiares el conocimiento tácito presenta los mismos problemas que en las no familiares; al no poder ser directamente apropiable ni transferido, esta característica se manifiesta con su uso en la actividad productiva.

Con respecto a la especialización, las empresas familiares presentan características particulares. La concentración de conocimiento especializa a los propietarios en su negocio particular, lo cual es consecuencia de las particularidades propias de este tipo de empresa, en la cual el conocimiento medular del negocio reside en el fundador-propietario o en un número limitado de integrantes de la familia. Esta concentración de conocimiento aumenta la consolidación del poder y el control, pero el intercambio limitado de experiencias puede disminuir la capacidad de las empresas familiares para crear nuevo conocimiento.

En líneas generales, la transferencia de conocimiento dentro de la misma empresa a través de las generaciones es un tema que puede explicar por qué la mayoría de las empresas familiares no sobreviven a la tercera generación, con una tasa de mortalidad mucho mayor durante la transición de la propiedad de la segunda generación. La marcha del predecesor o familiares clave podría resultar en una falta importante del know-how esencial para el éxito de la empresa familiar, siendo su transferencia de conocimientos de gran relevancia (Durst y Wilhelm, 2012). Por otro lado, la interferencia de los problemas familiares en la empresa puede generar una barrera para la comunicación que con el tiempo induce 
una menor transferencia de conocimiento, tanto tácito como explícito y por ende, entre otros aspectos, limitan los modos de conversión cruzado. Por otro lado, el sentido de compromiso, confianza, reputación, know-how, el fuerte sentido de identidad y un lenguaje familiar común les permite comunicarse más eficientemente e intercambiar mayor información con mayor privacidad (Barroso, 2013), favorecen los modos de conversión del conocimiento internos.

Transferir conocimiento es esencial para ayudar a promover buenas prácticas y proporciona oportunidades para el aprendizaje mutuo y la cooperación inter e intrageneracional, que a su vez estimula nuevo conocimiento (Marouf, 2007). En tal sentido, en las empresas familiares el conocimiento necesita ser acumulado entre los miembros de la familia para generar valor en el tiempo, en especial, cuando la nueva generación tiene que hacerse cargo del negocio. La transferencia de conocimiento de la generación anterior a la siguiente, es muy importante para poder dirigir bien la empresa, y a su vez, esta nueva generación tiene que añadir nuevo conocimiento y ofrecer nuevas perspectivas a la empresa familiar, al igual que también es necesario compartir conocimiento entre los integrantes de una misma generación

Este estudio ha abordado el nivel epistemológico del proceso de creación de conocimiento. Futuros estudios deberán centrarse en el nivel ontológico, partiendo del sistema de relaciones entre los diversos agentes creadores de conocimiento en la empresa familiar, así como en las relaciones causales que hipotéticamente pudieran darse entre estos dos niveles.

\section{Referencias Bibliográficas}

Ardichvili, Alexandre, (2008). Learning and Knowledge Sharing in Virtual Communities of Practice: Motivators, Barriers and Enablers. Advances in Developing Human Resources, Vol. 10, № 4 , pp 541-554.

Argyris, Chris. y Schön, D. (1978). Organizational learning: A theory of Action Perspective. Addison-Wesley, Mass.

Argyris, Chris y Schön, D. (1996). Organizational Learning II: Theory, Method and Practice, Addison-Wesley, Mass.

Arrow, Kenneth (1971). Essays in the Theory of Risk Bearing, Markham. Chicago, IL

Arrow, Kenneth (1984). "Information and Economic Behavior". In Collected Papers of Kenneth J. Arrow, Vol. 4, Cambridge, MA; Belknap Press.

Ayala C., Juan Carlos; Navarrete M., Ernesto: (2004). Efectos Tamaño y Sector sobre la Rentabilidad, Endeudamiento y Coste de la Deuda de las Empresas Familiares Riojanas. Cuadernos de Gestión. Vol. 4, № 1, pp. 35-53

Bañegil, Palacios Tomás; Barroso, Ascención y Tato J.L (2011). Profesionalizarse, Emprender y Aliarse para que la Empresa Familiar Continúe. Revista de Empresa Familiar; Vol. 1; № 2; pp 23-37.

Barney, Jay. (1991). Firm Resources and Sustained Competitive Advantage. Journal of Management. Vol. 17; № 1, pp 99-120.

Barney, Jay, y Arikan, Asly (2001). "The Resource-Based View: Origins and Implications", en Hitt, M. A.; Freeman, R. E. y Harrison, J. S. (eds.), Handbook of Strategic Management, Blackwell, Oxford, UK.

Barroso M, Ascención (2013). La Importancia de la gestión del Conocimiento en el Espíritu emprendedor de las Empre- 
sas Familiares. Tesis Doctoral. Universidad de Extremadura. España.

Bierly, Paul y Chakrabarti, Alok (1996). Generic Knowledge Strategies in the US Pharmaceutical Industry. Strategic Management Journal, Vol. 17(Winter Special Issue), pp123-135

Brenes, Esteban; Madrigal, Krissia y Molina, Germán (2006). Family Business Structure and Succession: Critical Topics in Latin American Experience. Journal of Business Research. Vol. 59; pp. 372-374.

Bueno, Eduardo (2002). Dirección Estratégica Basada en Conocimiento: Teoría y Práctica de la Nueva Perspectiva. En P. Morcillo (Coord.): Nuevas Claves para la Dirección Estratégica de la Empresa. Barcelona: Ariel, pp. 91-116.

Cabrera, María Katiuska (1998). Factores Determinantes del Éxito y Fracaso del Proceso de Sucesión en la Empresa Familiar. Tesis Doctoral, Universidad de las Palmas de Gran Canaria.

Clarke, J.L y Turner, P. (2005). Extending the Knowledge-based View: An Examination of Intellectual Property Strategies in Australian Biotechnology Firms Prometheus. Vol. 2; № 1; pp 85-100.

Chua, Jess; Chrisman Jay y Sharma, Pramodita (1999). Defining the Family Business by Behaviour Entrepreneurship Theory \& Practice, summer, pp 19-39.

Chua, Jess; Chrisman, James y Steier, Lloyd. (2003). Extending the Theoretical Horizons of Family Business Research Entrepreneurship Theory \& Practice. Summer, pp 331-338.

Cohen, Wesley y Levinthal, Daniel (1990). Absorptive Capacity: a New Perspective on Learning and Innovation. Administrative Science Quarterly, 128-152.

Comeche, José Manuel (2007). Influencia de los Factores Conductuales en la Capacidad de Acumulación, Genera- ción y Transmisión de Conocimientos en los Entrepreneurial team de la organización. En Decisiones basadas en el Conocimiento y en el Papel Social de la Empresa: XX Congreso Anual AEDEM, Vol. 1, pp 73.

Demsetz, Harold (1988). The Theory of the Firm Revisited, Journal of Law, Economics and Organization, Vol. 4, № 1 pp. 141-161.

Durst, Susan y Wilhelm, Stefan (2012). Knowledge Management and Sucession Planning in SMEs. Journal of Knowledge Management, Vol. 16, № 4, pp 637-649.

Endres, Megan Lee; Endres, Steven; Chowdhury, Sanjib; Alam, Intakjab (2007). Tacit Nowledge Sharing, Self Efficacy Theory and Application to the Open Source Community. Journal of Knowledge Management, Vol. 11, № 3, pp. 92-103.

Eisenhardt, Katleen y Martin, Jeffrey (2000). Dynamic Capabilities: What are they? Strategic Management Journal, Vol. 21, pp 1105-1121.

Gallo, Miguel A (1991). Ética de los Comportamientos Personales en la Empresa Familiar Documento de Investigación, № 372. IESE.

García A, María Ercilia; Lopez S, Jordy y Saldaña, Pilar (2002). Socialization Patterns of Successors in First to Second Generation Family Businesses, Family Business Review, Vol. XV, № 3, pp.189-203.

García A, María Ercilia (2001). Los Valores del Fundador y su Influencia en la Empresa Familiar en Galicia. Tesis Doctoral. Universidad de Barcelona. España.

Grant, Robert (1991). The Resource-Based Theory of Competitive Advantage: Implications for Strategic Formulation, California Management Review. Spring, pp. 114-135. 
Grant, Robert (1996). Toward a KnowledgeBased Theory of the Firm. Strategic Management Journal, Vol. 17 (winter special issue), pp 109-122.

Grant, Robert (1997). The Knowledge-Based View of the Firm: Implications for Management Practice. Long Range Planning, Vol. 30, № 3, pp 450-454.

Habbershon, Timothy y Williams, Mary (1999). A Resource-Based Framework for Assessing the Strategic Advantages of Family Firms, Family Business Review, Vol. 12, № 1, pp 1-25.

Handler Wendy (1989). Methodological Issues and Considerations in Studying Family Businesses. Family Business Review, Vol. 2, № 3, pp. 257-376.

Henderson, Rebecca y Cockburn, lain (1994). Measuring Competence? Exploring Firm Effects in Pharmaceutical Research. Strategic Management Journal, Vol. 15 (Winter Special Issue), pp 63-84.

Henry, Michael (2012). Tacit Knowledge Transfer in Family Business Succession. Doctoral Thesis. University of Southern Queensland. Canadá.

Hernández Fernández, Lisett (2008). La Gestión del Conocimiento en las Empresas Familiares. Colección de Textos Universitarios. Ediciones del Vicerrectorado Académico. Universidad del Zulia, Venezuela, pp110

Kim, Daniel (1993). The Link Between Individual and Organizational Learning. Sloan Management Review, fall, pp. 37-50.

Kogut, Bruce y Zander, Udo. (1992). Knowledge of the Firm, Combinative Capabilities, and the Replication of Technology. Organization Science, Vol. 3, № 3, pp 383-397.

Langlois, Richard y Everett, Michael (1984). ¿What is Evolutionary Economics? En: Evolutionary and Neo Shumpeterian Approache to Economics. Lars
Magnusson (ed) Business Economics Finances.

Lansberg, Irving (1983). Managing Human Resources in Family Firms: The Problem of Institutional Overlap. Organizational Dynamics, summer, pp 39-46.

Levin, Richard C; Klevorick, Alvin; Nelson, Richard R y Winter, Sidney G. (1987). Apropriating the Returns from Industrial Research and Development. Brookings Papers on Economic Activity, pp 783-820.

Lloria, María Begoña (2004). Diseño Organizativo, Facilitadores y Creación de Conocimiento. Un Estudio Empírico En Las Grandes Empresas Españolas, Tesis Doctoral. Universitat de Valencia, España.

López Sánchez, Mary Luz (2011). La Relevancia de la Gestión del Conocimiento en las Empresas. Apuntes del CENES, Vol. 30, № 51, pp 223-237.

Mahoney, Joseph y Pandian, Rajendran (1992). The Resource-Based View Within the Conversation of Strategic Management, Strategic Management Journal, Vol. 13, pp 363-380.

Marouf, Laila Naif (2007). Social Networks and Knowledge Sharing in Organizations: A Case Study. Journal of Knowledge Management, № 11:6, pp 110-125.

Morris, Michael; Williams Roy; Allen, Jeffrey y Avila, Ramón (1997). Correlates of Success in Family Business Transitions. Journal of Business Venturing, Vol. 12, pp 385-401.

Navarro, Ketty (2008). Estado Actual de la Investigación sobre Gestión del Conocimiento en Empresas Familiares. Revista de Ciencias Sociales, Vol. XIV, № 1, pp. 30-45.

Nelson, Richard; Winter, Sidney (1982) An Evolutionary Theory of Economic Change. Cambridge, MA: Harvard University Press. 
Nonaka, Ikujiro (1991). "The Knowledge-Creating Company". Harvard Business Review; Vol. 69, № 6, pp 96-04.

Nonaka, Ikujiro. y Takeuchi, Hirotaka (1995). The Knowledge-Creating Company: How Japanese Companies Create the Dynamics of Innovation, Oxford University Press, New YorkOxford.

Oltra, Víctor. (2002). "Influencia de las políticas de recursos humanos en los procesos de desarrollo y gestión del conocimiento". Tesis Doctoral. Universidad de Valencia.

Prahalad, C.V; Hamel, Gary. (1990). The Core Competence of the Corporation. Harvard Business Review LAD, pp 79-91.

Peteraf, Margaret (1993). "The Cornerstones of Competitive Advantage: A Resource-Based View", Strategic Management Journal, Vol. 14, pp 179-191.

Rus, Salvador y Rodríguez, María Pilar. (2005). "¿Qué es una Empresa Familiar?”, En Manual de la Empresa Familiar. Juan Corona (editor). Editorial Deusto. Barcelona, España.

Senge, Peter. (1990). The Fifth Discipline: The Art and Practice of the Learning Organization Oubleday/Currency,New York:

Sharma, Pramodita. (2004). An Overview of the Field of Family Business Studies: Currents Status and Directions for Future, Family Business Review, 12 (1), pp 1-36.

Simon, Herbert (1957). Models of Man: Social and Rational, John Wiley. New York:

Suehiro, A y N. Wailerdsak (2004). Family Business in Thailand. Its Management, Governance, and Future Challenges. ASEAN Economic Bulletin, Vol. 2, № 1, pp 81-93.
Schulze, William, Lubatkin, Michael y Dino, Richard (2003). Exploring the Agency Consquences of Ownership Dispersion Among the Directors of Private Family Firms. Academy of Management, Vol. 48, № 2, pp. 179-94.

Senge, Peter (1992). La Quinta Disciplina. Editorial Granica, Barcelona, España.

Spender, J.C y GranT, Robert. (1996). Knowledge and the Firm: Overview. Strategic Management Journal, Vol. 17, winter special issue, pp 5-9

Teece, David. (1986). Profiting from Technological Innovation: Implications for Integrations, Collaborations, Licensing and Public Policy. Research Policy, Vol. 15, pp 285-305.

Teece, David. J.; Pisano, Gary. y Shuen, Amy. (1997). Dynamic Capabilities and Strategic Management. Strategic Management Journal, Vol. 18, № 7, pp 509-533.

Trevinyo Rosa y Tapies, Josep (2010). Effective Knowledge Transfer in Family Firms. IESE, Working Paper 865, Business School, University of Navarra.

Vainrub, Roberto; Rodríguez, Aramís (2007). Empresas Familiares: Hallazgos obtenidos en un estudio realizado en 12 empresas familiares venezolanas. En Memorias del Segundo Seminario Anual de Investigación del Instituto de Investigaciones Económicas y Sociales, Universidad Católica Andrés Bello, Caracas 3 y 4 de noviembre de 2006 Editorial: IESA, Caracas, Venezuela.

Venter, Elmarie; Boshoff, Christo; y Maas, Gideon. (2005). The Influence of Successor-Related Factors on the Succession Process in Small and Medium-Sized Family Businesses, Family Business Review, Vol. 12, № 4, pp 283-303. 
Weick, Karl (1991). The nontraditional quality of organizational learning. Organization Science, Vol. 2, № 1, pp116-124.

WERNERFELT, Birger (1984). A Resource-Based View of the Firm. Strategic Management Journal, Vol. 5, № 2, pp 171-180.
WILLIAMS, Mary (2001). A belief-focused process model of organizational learning. Journal of Management Studies, Vol. 38, № 1, pp. 67-85

ZACK, Michael (1999). Managing Codified Knowledge. Sloan Management Review, Vol. 40, № 4, pp. 45-58. 